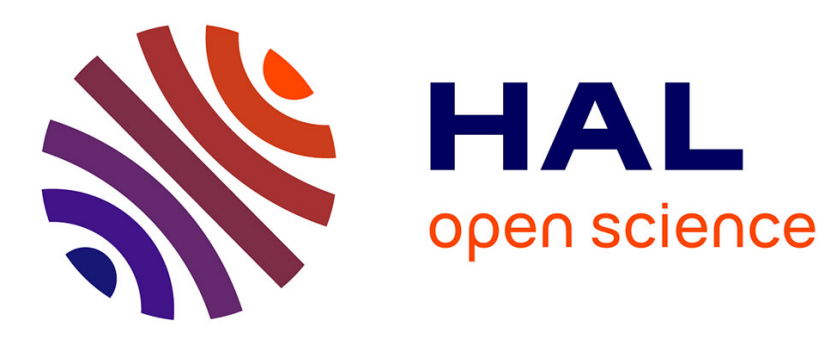

\title{
Enabling Communication Technologies for Medical Wireless Body-Area Networks
}

Oussama Haddad, Mohammad Ali Khalighi

\section{To cite this version:}

Oussama Haddad, Mohammad Ali Khalighi. Enabling Communication Technologies for Medical Wireless Body-Area Networks. Global LiFi Congress, Jun 2019, Paris, France. 10.1109/GLC.2019.8864122 . hal-02423690

\section{HAL Id: hal-02423690 https://hal.science/hal-02423690}

Submitted on 27 Mar 2020

HAL is a multi-disciplinary open access archive for the deposit and dissemination of scientific research documents, whether they are published or not. The documents may come from teaching and research institutions in France or abroad, or from public or private research centers.
L'archive ouverte pluridisciplinaire HAL, est destinée au dépôt et à la diffusion de documents scientifiques de niveau recherche, publiés ou non, émanant des établissements d'enseignement et de recherche français ou étrangers, des laboratoires publics ou privés. 


\title{
Enabling Communication Technologies for Medical Wireless Body-Area Networks
}

\author{
Oussama Haddad, Mohammad Ali Khalighi \\ Aix-Marseille University, CNRS, Centrale Marseille, Institut Fresnel, Marseille, France \\ Email: \{Oussama.Haddad, Ali.Khalighi\}@fresnel.fr
}

\begin{abstract}
The increasing percentage of aging population and chronic diseases on one hand, and the advances in the development of integrated short range wireless technologies on the other hand, have created a growing interest in the development of medical telemonitoring and telecare systems through the use of medical on-body sensor networks, also known as medical wireless body area networks (WBANs). This paper provides an overview of the main wireless technologies that can be used for WBANs in the medical domain and discusses the major requirements in such applications. While radio frequency technologies are well established solutions for interconnecting WBANs, the high risk of interference in such networks motivates the use of alternative or complementary technologies including optical wireless communications.
\end{abstract}

Index Terms-Wireless sensor networks; body area networks; medical on-body networks; optical wireless communications.

\section{INTRODUCTION}

According to the World Health Organization (WHO), Life expectancy is consistently increasing and this trend will continue thanks to more efficient medical follow-up of the newborns and a better epidemics prevention and control [1], [2]. At the same time, the changing lifestyle is resulting in an increased mortality rate due to cardiovascular diseases [3] as well as an increased number of chronic diseases such as diabetes [4]. These trends will put more and more pressure on the health-care systems, increasing the treatment costs for both health centers and patients.

With the advances made in the different fields of engineering, smart solutions can be developed to endorse traditional health-care methods. In particular, the advances in very-large-scale integration (VLSI) had made possible the integration of diverse sensors and micro-electromechanical systems (MEMS) in millimeter-size chips [5][7]. Furthermore, with the development of low-power shortrange communication technologies, wireless connection of these sensors and actuators can be readily done, giving rise to wireless sensor networks (WSNs), which are considered as the building blocks of the near-future Internetof-things (IoT) networks [8]. Indeed, the progress made in cloud computing and machine learning has made computing power and data analysis available at very low costs [9], which has, in turn, allowed removing the processing load from these WSNs.

The application of WSN technology to health-care has given birth to body sensor networks (BSNs) or wireless body-area networks (WBANs), which consist of networks of medical sensors located inside, on, or outside the human body. By the aid of such networks, early detection of health emergencies can be made possible. They can also be used for remote monitoring of vital signs of patients such as ECG, EEG, and temperature, as well as for remote drug delivery such as by insulin pumps. The obvious benefit of using such networks is in saving lives by more efficient and reactive monitoring and treatment of patients with chronic conditions and cost reduction. Compared with general WSNs, WBANs have specific characteristics including: smaller number of network nodes, necessity of a very small delay latency; non-stationary nature of the networks due to the movement of the patients; and heterogeneity of the type of transmitted signals (a mixture of continuous-time signals like ECG and event-based signals like temperature) [10].

A number of relatively recent works have studied different aspects of radio-frequency (RF) based WBANs. In [10], a survey of a few WBAN-related projects and enabling technologies at hardware and connectivity levels was presented. In [11], WBAN physical and data link layers were investigated, whereas the corresponding media-access control (MAC) and network layer protocols were discussed in [12] together with cross layer design. In [13], Movassaghi et al. presented in particular the different layers of the IEEE 802.15.6 standard dedicated to WBANs. Also, the requirements for wireless technologies that can be used for these networks were discussed in [14], and a survey on the related textile-based sensors was presented in [15].

Our aim in this paper is to present a general overview of the wireless technologies that are suitable for use in WBANs and to complement the previous works on these networks by considering other non-RF technologies including such ultrasound and optical wireless communications. After introducing the general applications of WBANs in Section II we discuss the architecture of these networks in Section III. The major requirements of WBANs for medical applications are specified in Section IV. We then focus on the wireless technologies for WBANs in Section V including RF, ultrasonic and optical wireless, with a special focus on the latter, and specify some related recent works. Lastly, Section VI concludes the paper.

\section{WBANS FOR MEDICAL APPLICATIONS}

The use of WBANs has been considered for different applications including medical, military, sport, interactive gaming, etc. Here we focus on the main applications of these networks in the medical domain. 


\section{A. Vital sign monitoring}

WBANs can be used for continuous monitoring of vital signs such as electrocardiogram (ECG), electroencephalogram (EEG), electromyogram (EMG), blood glucose level, blood pressure, heart rate, body temperature, motion, etc. The related physiological signals can be used to monitor or diagnose diseases such as cardiovascular disease, diabetes, epilepsy, as well as for monitoring, sleep, stress, activity, etc. Some typical relevant physiological sensors include: ECG sensors to record the heart electrical activity, i.e., the electrical changes that arise from the pattern of polarizing and depolarizing of the heart muscle; EEG sensors to record the brain electrical activity using several electrodes placed along the scalp to measure voltage fluctuations resulting from ionic currents within the neurons; EMG sensors to record the electrical activity produced by skeletal muscles by measuring the electric potential generated by muscle cells when electrically or neurologically activated; and pulse oximetry (or SO2) sensor to measure the blood oxygen saturation based on the changing absorbance of two wavelengths of light passing through the body and determining the absorbance corresponding to the pulsing arterial blood.

Continuous monitoring of these vital signs helps also to detect health emergencies and to alarm physicians and health practitioners for early interventions, especially in the case of elderly people and disabled persons.

\section{B. Therapeutic stimulation}

Another important application of WBANs is to deliver therapeutic treatments via actuators that work through electrical stimulation or biochemical injections. Typical examples are: pacemakers that deliver electrical impulses through electrodes to regulate the electrical conduction of the heart; cochlear implants that is used to capture sound using a microphone and to convert it to an electrical signal to stimulate directly the auditory nerve; neuromuscular stimulators that deliver electrical impulses muscles, mimicking the action of the central nervous system; and insulin pump used for administering the delivery of insulin based on the blood glucose level. Some other examples include defibrillator, brain stimulator, artificial retina, etc.

\section{ARCHITECTURE OF A WBAN}

The general architecture of a WBAN consists of three different tiers: intra-WBAN with a typical transmission range one meter or so, extra-WBAN, and beyond-WBAN communications [13], as illustrated in Fig. 1. For the first, i.e., intra-WBAN tier, communication is between sensor nodes on or inside the body on one hand, and a coordinator node on the other hand. Sensor nodes can be either sensors that measure physiological data, or actuators that upon feedback information from sensors, conduct an electrical or biochemical stimulation. As concerns extra-WBAN tier, communication takes place between the coordinator node and one or more access points (APs), which can be part of the infrastructure or be placed in an ad-hoc mode. After collecting data from different sensor nodes, the coordinator node transmit it to the AP, probably after data fusion or

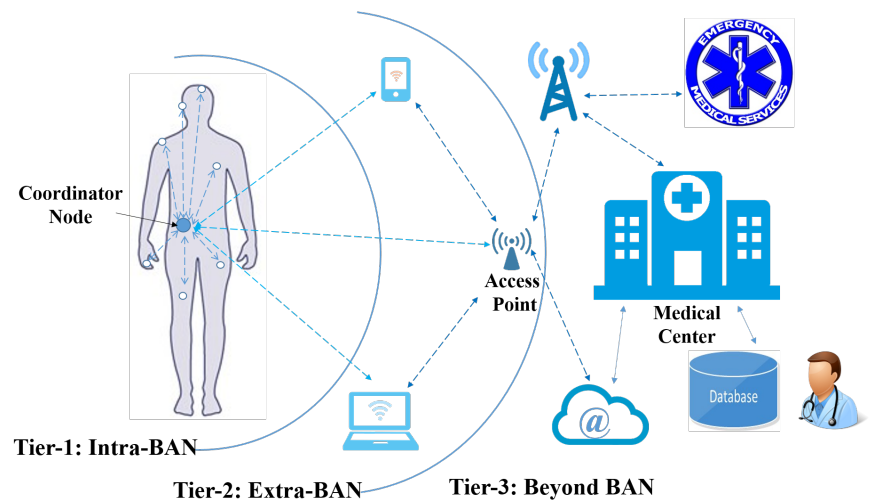

Fig. 1: A typical WBAN with the underlying communication tiers.

compression. The communication between the AP and the medical server concerns the third tier, that is done via Internet or a cellular network. The received data could be stocked in a database and/or used by the medical staff for real-time diagnosis or monitoring.

\section{SPECIFIC REQuiREMENTS of MedicAl WBANs}

As mentioned previously, a WBAN used in medical applications, has special requirements that differentiate it from other networks. We briefly present these considerations in the following.

\section{A. Data rate and link reliability}

Based on the targeted application, nodes in a WBAN have different requirement in terms of data rate, which can range from a few bits per second (bps), e.g. for a temperature sensor, to a few Mbps, e.g., for capsule endoscopy. According to the IEEE 802.15.6 standard requirements, WBAN links have to support data rates in the range of $10 \mathrm{Kbps}$ to $10 \mathrm{Mbps}$ [16]. Meanwhile, the network should satisfy requirements in terms of quality-of-service (QoS) to ensure reliable communication, which is usually specified in terms of bit-error-rate (BER). The BER requirement depends on the medical device and can vary from $10^{-3}$ for deep brain stimulation to $10^{-10}$ for EEG recording. Also, the required delay latency for medical applications is less than $125 \mathrm{~ms}$ [16]. Table 1 presents typical data-rate and BER requirements for some WBAN applications [17].

\section{B. Energy consumption}

Obviously, low energy consumption is an important feature of WBANs because it affects the battery lifetime of the medial devices. Battery lifetime is in particular crucial for implant devices where replacing power sources needs a costly surgical operation, although ongoing research considers harvesting techniques to recharge the device batteries remotely [18], [19].

Moreover, a high energy consumption may cause a temperature rise in the tissue that surrounds the sensor. As the human tissue is semi-conductive, it will absorb a part of the radiations emitted at transmission, which can cause severe thermal damage to sensitive organs. The amount of energy absorption is defined by the so-called specific absorption 
TABLE I: Requirements on data-rate and BER for some medical applications [20].

\begin{tabular}{|c||c||c|}
\hline \multicolumn{1}{|c||}{ Sensor nodes } & Target data rate & Target BER \\
\hline ECG & $72 \mathrm{Kbps}$ & $<10^{-10}$ \\
\hline EEG & $86.4 \mathrm{Kbps}$ & $<10^{-10}$ \\
\hline EMG & $1.536 \mathrm{Mbps}$ & $<10^{-10}$ \\
\hline SPO2 & $32 \mathrm{bps}$ & $<10^{-10}$ \\
\hline Temperature, glucose level, pH & $20 \mathrm{bps}$ & $<10^{-10}$ \\
\hline Cochlear implant & $\sim 200 \mathrm{Kbps}$ & $<10^{-10}$ \\
\hline Deep brain stimulation & $128-320 \mathrm{Kbps}$ & $<10^{-3}$ \\
\hline Accelerometer, blood pressure & $<10 \mathrm{Kbps}$ & $<10^{-10}$ \\
\hline Endoscope capsule & $1 \mathrm{Mbps}$ & $<10^{-10}$ \\
\hline
\end{tabular}

rate (SAR) that needs to be minimized for WBAN sensors. The upper limit of SAR exposure is fixed to $2 \mathrm{~W} / \mathrm{Kg}$ over $10 \mathrm{~g}$ of tissue in the European Union countries and Japan, and $1.6 \mathrm{~W} / \mathrm{Kg}$ over $1 \mathrm{~g}$ of tissue in the United States and Australia, which are equivalent to maximum transmit powers of 20 and $1 \mathrm{~m}$, respectively [16].

\section{Security and privacy}

Security is of high concern in medical WBANs since medical data is considered as sensitive, private, and confidential. As such, data should be encrypted to ensure user's privacy, and authentication mechanisms should be used to verify data integrity and to ensure that data is being sent from a trusted node. This is an important issue in RF-based networks where data-carrying signals can be intercepted and jammed relatively easily, as we will discuss later.

\section{EXISTING WiRELESS TECHNOLOGIES FOR WBANS AND RELATED WORKS}

\section{A. RF technologies}

Most of the current realizations of WBAN systems are based on the rather well-known wireless personal-area network (WPAN) technologies (IEEE 802.15) operating in the $2.4 \mathrm{GHz}$ ISM (Industrial, Scientific, and Medical) band. Bluetooth as a popular short-range communication system [21] with two main modes of basic rate / enhanced data-rate (BR/EDR) and low-energy (LE), has received particular attention. It is especially the case for the fourth version, known as Bluetooth low-energy (BLE), designed for device-to-device communications with low complexity, low cost, and low power consumption, offering data rates up to 2 Mbps. The most recent (i.e., the fifth) version of Bluetooth, specially developed for IoT device connectivity, has different power classes that can serve different ranges of 100,10 , and $1 \mathrm{~m}$, with a maximum transmit power of $100,2.5$, and $1 \mathrm{~mW}$, respectively.

Another solution is the IEEE 802.15.4 standard [22], which was developed for low-cost wireless connectivity for applications with limited power and relaxed throughput requirements. With a simple and flexible protocol, it offers data rates from 20 to $250 \mathrm{Kbps}$ within a typical range of $10 \mathrm{~m}$ and a transmit power limited to $10 \mathrm{~mW}$. The popular Zigbee [23] is a low-cost and low-power technology built on the top of IEEE 802.15.4 standard by modifying the network and application layers.

Lastly, the short-range low-power IEEE 802.15.6 standard
[24] was specifically designed for WBANs, offering up to 15 Mbps data-rate with a transmit power between 0.1 and $1 \mathrm{~mW}$. It has been developed to overcome the limitations of the other existing WPANs to meet the requirements of medical applications discussed in Section IV. This standard defines narrow-band transmission in the ISM, WMTS (Wireless Medical Telemetry Service), and MICS (Medical Implant Communication Service) bands, as well as ultrawideband (UWB) and human body communication (HBC), with data-rates in the range of $57.5-971.4 \mathrm{Kbps}$ for narrow-band transmission, $0.487-15.6 \mathrm{Mbps}$ for UWB, and $164 \mathrm{Kbps}-1.3 \mathrm{Mbps}$ for $\mathrm{HBC}$ (operating at $21 \mathrm{MHz}$ ).

Although Bluetooth and Zigbee do not meet the requirements in terms of data-rate, reliability, and SAR of certain medical applications (e.g., in hospital environments) [20], most of the reported WBAN implementations rely on these standards [25]-[30]. However, to the best of the authors' knowledge, no realization of WBANs has been reported based on IEEE 802.15.6, which can be explained by the availability of Bluetooth and Zigbee transceivers and their ease of use compared to the former. For instance, a system for monitoring temperature and heart rate was proposed in [27] based on Power-Class 2 (with a maximum emitted power of $2.5 \mathrm{~mW}$ ) EDR Bluetooth module. In [26] a 3-lead ECG system was designed to measure the respiration rate, based on a Power-Class 2 BLE module. A similar device was used in [31] for a wearable WBAN with solar energy harvesting to measure body temperature, heart rate, and fall detection.

Concerning Zigbee applications, a wearable system was designed in [30] comprising of an accelerometer and temperature and humidity sensors, using an IEEE 802.15.4 compatible transceiver capable of implementing Zigbee protocols and transmitting at $2 \mathrm{~mW}$. Also, a wearable system equipped with GPS and 3-lead ECG was proposed in [32] for the purpose of fall detection and assisting elderly people. A health monitoring system equipped with a 1lead ECG, a pulse sensor, and a body weight sensor was also designed in [33] where a Zigbee transceiver module with $6.3 \mathrm{~mW}$ was used to interconnect sensors with a coordinator node, and a Bluetooth v.2 module with $2.5 \mathrm{~mW}$ to connect this latter to an AP.

\section{B. Ultrasonic technologies}

Ultrasonic communications are enabled by the propagation of acoustic waves inside the human body at frequencies higher than $20 \mathrm{KHz}$. They have the potential to complement or replace RF technologies for implant communications thanks to their low attenuation in the human tissues. Transceivers used for ultrasonic communications are mainly piezoelectric transducers. In [34], an implantable pressure sensor was tested with a data-rate of $40 \mathrm{Kbps}$ and a transmit power of $100 \mu \mathrm{W}$ through a $12 \mathrm{~cm}$ tick castor oil. Also, in [35] an ultrasonic wideband system communicating through a human kidney phantom at $10 \mathrm{~cm}$ was demonstrated with a data-rate of $700 \mathrm{Kbps}$ and a power consumption of $40 \mu \mathrm{W}$ at a BER of $10^{-6}$. 


\section{Optical wireless technologies}

Optical wireless communications (OWC) in the infrared (IR), visible, or ultra-violet parts of the spectrum are a potential alternative or complement to RF technologies for medical WBANs thanks to their high immunity to external interference and their inherent security due to confinement in indoor spaces. In addition, the transmit power in OWC systems is not constrained by SAR regulations like for RF counterparts. The interest of the optical wireless technologies has been investigated in several works for on-body, extra-body, and in-body medical applications.

The use of OWC for on-body WBAN links was investigated in [17], where their practical feasibility was demonstrated. Characterizing the communication channel using ray-tracing based numerical simulations, the authors evaluated the theoretical performance of an optical link using on-off-keying modulation and code-division multipleaccess for connecting several sensors for the case of purely diffuse links (i.e., without a line-of-sight). They showed a BER of $10^{-10}$ for data-rates from 10 to $890 \mathrm{Kbps}$, with a power consumption of $17 \mathrm{~mW}$ for $10 \mathrm{Kbps}$ data-rate, which is much lower than the maximum permitted emitted power for IR communications (based on eye safety regulations) [17]. Lower power consumption would be attainable using more efficient transmission schemes, however.

Concerning extra-body links, a bidirectional OWC system was studied in [36] for connecting a WBAN coordinator node with an AP, where visible-light communication (VLC) was used for downlink (the AP being integrated into a LED luminary, for instance) and IR transmission for uplink. It was shown that for most medical sensors, it is possible to satisfy QoS and data-rate requirements with a low radiated power for both IR and VLC links. Moreover, the use of OWC for transmitting accelerometer data for indoor physical activity monitoring was investigated in [37] where its efficacy was demonstrated through experimental measurements using a designed wearable system. On the other hand, in-body communication in the optical domain has attracted increasing attention in the past few years as it provides a better performance in terms of data rate and interference level, as compared to RF technologies [38][40]. For instance, a transdermal optical link operating at $1 \mathrm{Mbps}$ through porcine skin was reported in [38] using a $860 \mathrm{~nm}$ LED at the transmitter and a PIN photo-diode at the receiver. Also, a telemetry system based on a laser diode was studied in [41] with a data rate of $16 \mathrm{Mbps}$ through a skin thickness of $4 \mathrm{~mm}$ and a power consumption of less than $10 \mathrm{~mW}$. Experimental results were also presented in [42] for data transmission through a $1 \mathrm{~mm}$ thick chicken derma, where a data rate of $20 \mathrm{Kbps}$ at a BER of $10^{-6}$ was reported with both direct and retroflection links with a power consumption of $4 \mathrm{~mW}$ and $0.4 \mu \mathrm{W}$, respectively. Using a VCSEL laser, a transmission link through a $4 \mathrm{~mm}$ tissue was demonstrated in [43], achieving $50 \mathrm{Mbps}$ data rate at BER of $10^{-5}$ with a power consumption of $4.1 \mathrm{~mW}$. An extension of this study was done in [44], where a data rate of $100 \mathrm{Mbps}$ at a BER of $2 \times 10^{-7}$ was achieved with a power consumption of $2.1 \mathrm{~mW}$ through a tissue of an anesthetized sheep of $2.5 \mathrm{~mm}$ thickness. These results were further extended in [45] to a bidirectional link through a $2 \mathrm{~mm}$ porcine skin, achieving $1 \mathrm{Mbps}$ with a power consumption of $290 \mu \mathrm{W}$ in downlink and $100 \mathrm{Mbps}$ with $3.2 \mathrm{~mW}$ in uplink.

\section{CONCLUSIONS}

In this paper, we have provided a review of WBANs for medical applications, the typical medical sensors that can be used in such networks, the particular requirements that distinguish WBANs from other personal networks, and more importantly, the enabling wireless technologies with a special focus on the OWC-based techniques. With the increasing demand for medical telemonitoring and telecare as well as more efficient hospital systems, the use of WBANs attracts more and more attention. The optical wireless technology appears to be a promising solution in such applications thanks to its immunity to RF interference and inherent security, in particular. We reviewed several proofsof-concept based on optical wireless data transmission with high data-rate and low power consumption.

\section{ACKNOWLEDGMENT}

This work is supported by VisIoN, a European project funded by the European Union's Horizon 2020 research and innovation program under the Marie Skłodowska-Curie Grant Agreement No. 764461.

\section{REFERENCES}

[1] "WHO article: Life expectancy and healthy life expectancy data by country," http://apps.who.int/gho/data/node.main.688?lang=en, accessed: 16-02-2019.

[2] "WHO article: Life expectancy increased by 5 years since 2000," https://www.who.int/en/news-room/detail/19-05-2016-lifeexpectancy-increased-by-5-years-since-2000-but-health-inequalitiespersist, accessed: 16-02-2019.

[3] C. X. Wong, A. Brown, D. H. Lau, S. S. Chugh, C. M. Albert, J. M Kalman, and P. Sanders, "Epidemiology of sudden cardiac death Global and regional perspectives," Heart, Lung and Circulation, vol. 28 , no. 1, pp. 6-14, Jan. 2019.

[4] "WHO article: Diabetes," https://www.who.int/news-room/factsheets/detail/diabetes, accessed: 16-02-2019.

[5] Y. Ma, Z. Luo, C. Steiger, G. Traverso, and F. Adib, "Enabling deep-tissue networking for miniature medical devices," in ACM SIGCOMM Conference, Aug. 2018, pp. 417-431, Budapest, Hungary.

[6] P. Abiri, A. Abiri, R. R. S. Packard, Y. Ding, A. Yousefi, J. Ma, M. Bersohn, K.-L. Nguyen, D. Markovic, S. Moloudi, and T. K Hsiai, "Inductively powered wireless pacing via a miniature pacemaker and remote stimulation control system," Scientific Reports, vol. 7, no. 6180, 2017.

[7] J. Kim, A. Balankutty, R. Dokania, A. Elshazly, H. S. Kim, S. Kundu, S. Weaver, K. Yu, and F. O'Mahony, "A 112Gb/s PAM-4 transmitter with 3-tap FFE in 10nm CMOS," in IEEE ISSCC Conference, Feb. 2018, pp. 102-104, San Francisco, CA.

[8] L. Atzori, A. Iera, and G. Morabito, "The Internet of Things: A survey," Computer Networks, vol. 54, no. 15, pp. 2787 - 2805, 2010.

[9] B. Varghese and R. Buyya, "Next generation cloud computing: New trends and research directions," Future Generation Computer Systems, vol. 79, pp. $849-861,2018$.

[10] H. Cao, V. Leung, C. Chow, and H. Chan, "Enabling technologies for wireless body area networks: A survey and outlook," IEEE Communications Magazine, vol. 47, no. 12, pp. 84-93, Dec. 2009.

[11] M. Chen, S. Gonzalez, A. Vasilakos, H. Cao, and V. C. Leung, "Body area networks: A survey," Mobile Networks and Applications, vol. 16, no. 2, pp. 171-193, Apr. 2011.

[12] B. Latré, B. Braem, I. Moerman, C. Blondia, and P. Demeester, "A survey on wireless body area networks," Wireless Networks, vol. 17, no. 1, pp. 1-18, Jan. 2011. 
[13] S. Movassaghi, M. Abolhasan, J. Lipman, D. Smith, and A. Jamalipour, "Wireless body area networks: A survey," IEEE Communications Surveys \& Tutorials, vol. 16, no. 3, pp. 1658-1686, Jan 2014.

[14] M. Ghamari, B. Janko, R. S. Sherratt, W. Harwin, R. Piechockic, and C. Soltanpur, "A survey on wireless body area networks for eHealthcare systems in residential environments," Sensors, vol. 16, no. 6, June 2016.

[15] S. Majumder, T. Mondal, and M. J. Deen, "Wearable sensors for remote health monitoring," Sensors, vol. 17, no. 1, pp. 261-268, Jan. 2017.

[16] B. Zhen, M. Patel, S. Lee, E. Won, and A. Astrin, "TG6 technical requirements document (TRD), IEEE P802.15-08-0644-09-0006,' Sept. 2008

[17] A. Julien-Vergonjanne, S. Sahuguède, and L. Chevalier, Optical Wireless Communications: An Emerging Technology. Springer, 2016, ch. Optical Wireless Body Area Networks for Healthcare Applications, pp. 569-587.

[18] X. Pu, W. Hu, and Z. L. Wang, "Toward wearable self-charging power systems: The integration of energy-harvesting and storage devices," Small, vol. 14, no. 1, Jan. 2018.

[19] P. D. Diamantoulakis and G. K. Karagiannidis, "Simultaneous lightwave information and power transfer (SLIPT) for indoor IoT applications," in GLOBECOM Conference, Dec. 2017, pp. 1-6, Singapore, Singapore.

[20] D. Lewis, "TG6 applications summary, IEEE P802.15-08-0407-03," Sept. 2008.

[21] Bluetooth Core Specification Version 5.1, IEEE 802.15.6 Std., Jan. 2019.

[22] IEEE Standard for Low-Rate Wireless Networks, IEEE 802.15.6 Std., Apr. 2016.

[23] ZigBee Document 053474r20, IEEE 802.15.6 Std., Sept. 2012.

[24] IEEE Standard for Local and Metropolitan Area Networks - Part 15.6: Wireless Body Area Networks, IEEE 802.15.6 Std., Feb. 2012.

[25] M. Esposito, A. Minutolo, R. Megna, M. Forastiere, M. Magliulo, and G. D. Pietro, "A smart mobile, self-configuring, context-aware architecture for personal health monitoring," Engineering Applications of Artificial Intelligence, vol. 67, pp. 136 - 156, Jan. 2018.

[26] J. Wannenburg, R. Malekian, and G. P. Hancke, "Wireless capacitivebased ECG sensing for feature extraction and mobile health monitoring," IEEE Sensors Journal, vol. 18, no. 14, pp. 6023-6032, July 2018.

[27] A. Ahmed, A. A. Lukman, A. James, O. O. Mikail, B. U. Umar, and E. Samuel, "Human vital physiological parameters monitoring: A wireless body area technology based Internet of things," Jurnal Teknologi dan Sistem Komputer, vol. 6, no. 3, pp. 115-121, July 2018.

[28] N. Dey, A. S. Ashour, F. Shi, S. J. Fong, and R. S. Sherratt, "Developing residential wireless sensor networks for ECG healthcare monitoring," IEEE Transactions on Consumer Electronics, vol. 63 , no. 4, pp. 442-449, Nov. 2017.

[29] M. Clarke, J. de Folter, V. Verma, and H. Gokalp, "Interoperable end-to-end remote patient monitoring platform based on IEEE 11073 PHD and ZigBee health care profile," IEEE Transactions on Biomedical Engineering, vol. 65, no. 5, pp. 1014-1025, May 2018.

[30] A. M. Cristiani, G. M. Bertolotti, E. Marenzi, and S. Ramat, "An instrumented insole for long term monitoring movement, comfort, and ergonomics," IEEE Sensors Journal, vol. 14, no. 5, pp. 1564 1572, May 2014.

[31] T. Wu, F. Wu, J. Redouté, and M. R. Yuce, "An autonomous wireless body area network implementation towards IoT connected healthcare applications," IEEE Access, vol. 5, pp. 11413-11422, July 2017.

[32] L. Wang, Y. Hsiao, X. Xie, and S. Lee, "An outdoor intelligen healthcare monitoring device for the elderly," IEEE Transactions on Consumer Electronics, vol. 62, no. 2, pp. 128-135, May 2016.

[33] K. Becher, C. P. Figueiredo, C. Mühle, R. Ruff, P. M. Mendes, and K. . Hoffmann, "Design and realization of a wireless sensor gateway for health monitoring," International Conference of the IEEE Engineering in Medicine and Biology, pp. 374-377, Aug. 2010 , Buenos Aires, Argentina.

[34] M. J. Weber, Y. Yoshihara, A. Sawaby, J. Charthad, T. C. Chang, R. Garland, and A. Arbabian, "A high-precision $36 \mathrm{~mm} 3$ programmable implantable pressure sensor with wully ultrasonic powerup and data link," in Symposium on VLSI Circuits, June 2017, pp. C104-C105, Kyoto, Japan.

[35] G. E. Santagati and T. Melodia, "Experimental evaluation of impulsive ultrasonic intra-body communications for implantable biomedical devices," IEEE Transactions on Mobile Computing, vol. 16, no. 2 , pp. 367-380, Feb. 2017.

[36] C. Le Bas, S. Sahuguède, A. Julien-Vergonjanne, P. Combeau, and L. Aveneau, "Infrared and visible links for medical body sensor networks," in Global LiFi Congress (GLC), Feb. 2018, pp. 1-6, Paris France.

[37] C. Le Bas, S. Sahuguede, and A. Julien-Vergonjanne, "Theoretical and experimental approach for the design of an optical wireless physical activity monitoring system," International Journal of Wireless Information Networks, vol. 24, no. 2, pp. 65-77, June 2017.

[38] J. L. Abita and W. Schneider, "Transdermal optical communications," John Hopkins APL Tech, vol. 25, no. 3, pp. 261-268, 2004.

[39] M. Faria, L. N. Alves, and P. S. de Brito Andre, Visible Light Communications: Theory and Applications. CRC-Press, 2017, ch. Transdermal Optical Communications, pp. 309-336.

[40] S. E. Trevlakis, A.-A. A. Boulogeorgos, P. C. Sofotasios, S. Muhaidat, and G. K. Karagiannidis, "Optical wireless cochlear implants," Biomedical Optics Express, vol. 10, no. 2, pp. 707-730, Feb. 2019

[41] S. Parmentier, R. Fontaine, and Y. Roy, "Laser diode used in 16 $\mathrm{Mb} / \mathrm{s}, 10 \mathrm{mw}$ optical transcutaneous telemetry system," in IEEE Biomedical Circuits and Systems Conference, Nov. 2008, pp. 377380, Baltimore, MD.

[42] Y. Gil, N. Rotter, and S. Arnon, "Feasibility of retroreflective transdermal optical wireless communication," Applied Optics, vol. 51, no. 18 , pp. 4232-4239, June 2012.

[43] T. Liu, U. Bihr, S. M. Anis, and M. Ortmanns, "Optical transcutaneous link for low power, high data rate telemetry," in International Conference of the IEEE Engineering in Medicine and Biology Society, Aug. 2012, pp. 3535-3538, San Diego, CA.

[44] T. Liu, U. Bihr, J. Becker, J. Anders, and M. Ortmanns, "In vivo verification of a 100 Mbps transcutaneous optical telemetric link," in IEEE BioCAS Conference, Oct. 2014, pp. 580-583, Lausanne, Switzerland.

[45] T. Liu, "Bidirectional optical transcutaneous telemetric link for brain machine interface," Electronics Letters, vol. 51, no. 2, pp. 1969 1971, Nov. 2015 